Magn Reson Imaging Clin N Am. 2017 May ; 25(2): 231-243. doi:10.1016/j.mric.2017.01.002.

\title{
Principles of Simultaneous PET/MR Imaging
}

\author{
Ciprian Catana, MD, PhD [Associate Professor] \\ Radiology, Athinoula A. Martinos Center for Biomedical Imaging, Department of Radiology, \\ Massachusetts General Hospital and Harvard Medical School, Charlestown, MA
}

\begin{abstract}
Keywords
PET; MRI; PET/MRI; simultaneous imaging; multimodal imaging; integrated hardware; MRbased attenuation correction; time-of-flight PET
\end{abstract}

\section{Introduction}

Combining positron emission tomography (PET) and magnetic resonance imaging (MRI) into a single device capable of simultaneously acquiring both datasets has been of interest for several decades, even before the emergence (and eventual clinical success) of integrated PET and computed tomography (PET/CT) scanners (5). This was motivated by the complementary nature of the information provided by these two powerful imaging modalities that are currently widely used for research and clinical applications. On one hand, PET can assay with very high molecular sensitivity a wide range of biological processes (from tracking the in vivo distribution of small molecules to cells) using radionuclides that decay by positron emission. However, PET has limited spatial resolution and most often the images lack the anatomical detail required for clinical interpretation. On the other hand, MRI provides high spatial resolution anatomic images with excellent soft tissue contrast even using routine techniques. More advanced MR sequences exploit other sources of endogenous contrast to study physiology (e.g. water diffusion), function (e.g. blood oxygenation level dependent contrast) and metabolism (e.g. relative concentration of various metabolites), while passive contrast agents (e.g. gadolinium, iron oxide) that modify the tissue relaxation times can further enhance contrast in the resulting images. The main limitations of MRI in this context are that its sensitivity is several orders of magnitude smaller than that of PET and the lack of absolute quantification.

CORRESPONDING AUTHOR. Ciprian Catana.

AUTHOR CONTACT INFORMATION

Ciprian Catana, Athinoula A. Martinos Center for Biomedical Imaging, Massachusetts General Hospital, Bld. 149, 13th St, Room 2.301, Charlestown, MA 02129, ccatana@nmr.mgh.harvard.edu

Publisher's Disclaimer: This is a PDF file of an unedited manuscript that has been accepted for publication. As a service to our customers we are providing this early version of the manuscript. The manuscript will undergo copyediting, typesetting, and review of the resulting proof before it is published in its final citable form. Please note that during the production process errors may be discovered which could affect the content, and all legal disclaimers that apply to the journal pertain.

DISCLOSURE STATEMENT

The Author has nothing to disclose. 
While software co-registration or a side-by-side analysis can be performed to "fuse" the PET and MRI data acquired separately, this approach eliminates the possibility of temporally correlating the measurements, which could revolutionize a number of research and clinical applications. Furthermore, in those patients requiring both examinations, the simultaneous acquisition could improve the spatial registration between the two images, the workflow and increase the patient's comfort. For these reasons, we will discuss in this article only those aspects that are relevant to simultaneous acquisition.

\section{Technical aspects that have to be considered for integrating PET and MRI CONSIDERATIONS ON THE PET SIDE}

The major technical challenge from the PET perspective was that photomultiplier tubes (PMTs), used until recently in virtually all commercially available PET scanners, are very sensitive to even small magnetic fields and cannot be operated inside modern preclinical or clinical high-field MR devices. To overcome this limitation in the early days of PET/MRI, long optical fibers were used to channel the light from the scintillator crystals placed inside the MR system to PMTs located at a safe distance (6). Several generations of small animal imaging devices have subsequently been built using PMTs in an effort to increase the PET performance in terms of the spatial resolution uniformity $(7,8)$ and axial coverage $(9-12)$. All these efforts required substantial modification of the PET detectors to maximize compatibility with existing MRI devices. Alternatively, minimally modified PET detectors have been integrated in specialized MRI scanners (e.g. split-magnet (13) or field-cycled (14, $15)$ ).

In spite of this very important pioneering work, PET/MRI would have likely failed to transition from small animal to human imaging if it were not for the emergence of MRcompatible photon detector technology that allowed the placement of the PET detectors inside the magnet's bore. Fortunately, solid-state photon detectors have reached a level of maturity that allowed them to replace PMTs for this application. Avalanche photodiodes (APDs) were the first such photon detectors that were demonstrated to work even inside ultra-high-field magnets (16) and were used to build MR-compatible PET inserts for small animal imaging (17-19). More importantly, this technology allowed Siemens to build the first PET/MRI prototype scanner for human brain imaging (20). This device was called BrainPET and was designed to fit into the bore of the standard 3T whole body MRI scanner (Magnetom Trio, Siemens, Erlangen, Germany). Although only a handful of BrainPET prototypes were ever built and only three of them are still in use, these inserts have been used for many studies ranging from those aimed at investigating the mutual interference between the two devices and the performance of the APD-based PET detector technology $(21,22)$, developing methods to use the information obtained from one device to improve the other modality (23-26), and demonstrating the tremendous potential of PET/MRI through proof-of-principle studies in small animal, non-human primates and humans (27$35)$.

Additional challenges such as the need to carefully shield the PET detectors to minimize the electromagnetic interference with the MR system or to control for temperature variations in 
the detectors caused by the eddy currents induced by the switching MR gradients had to be considered when using these solid-state photon detectors.

More recently, Geiger mode APDs (also called solid-state photomultipliers (SSPM), silicon photomultipliers (SiPMs) or multi-photon pixel counters (MPPC)) have emerged as very promising candidates for replacing APDs as the photon detector of choice for simultaneous PET/MR imaging and several groups have developed small animal scanners using this technology (36-41). There are also now companies (e.g. Cubresa, Inc., Bruker Biospin) that offer MR-compatible PET inserts designed to operate inside existing small animal MRI devices.

\section{CONSIDERATIONS ON THE MR SIDE}

Simultaneous acquisition requires the placement of PET components (e.g. scintillators, photon detectors and associated electronics, cooling components, shielding material, etc.) in the bore of the MR scanner. This is a challenge because the main magnetic field homogeneity needs to be maintained for proper MR operation. For this reason, nonmagnetic versions of even standard components (e.g. resistors, capacitors, connectors, etc.) have to be used especially when such components are to be placed close to the subject. Similarly, scintillator materials with a magnetic susceptibility close to that of human tissue (i.e. lutetium-based scintillators instead of lutetium gadolinium oxyorthosilicate) are preferred (42).

Electromagnetic interference in the radiofrequency part of the spectrum has to be minimized to avoid introducing artifacts in the MR images. This is particularly challenging when PET photon detectors and electronics need to be integrated with the MR scanner as they contain radiofrequency-radiating components. The shielding required to minimize the interference is of special concern because eddy currents can be induced in the shielding enclosures by the changing gradients potentially leading to image artifacts and spatial distortions.

To enable simultaneous acquisition, the MR radiofrequency coils have to be positioned in the PET field of view and thus contribute to photon attenuation. To maximize their PET compatibility, they have to be carefully designed to minimally attenuate the $511 \mathrm{keV}$ photons and any changes have to be made in a way that maintains their performance.

The diameter of the bore of most commercially available MR scanners is no larger than 60 $\mathrm{cm}$, which means there is minimal space left to integrate PET components. Fortunately, larger $70 \mathrm{~cm}$ diameter bore scanners have recently been introduced by all the three major medical equipment manufacturers (i.e. Siemens, General Electric and Philips) and the integrated PET/MR scanners discussed in the next section were built starting from such systems.

\section{Integrated PET/MRI hardware for whole-body human imaging SIEMENS BIOGRAPH mMR}

Siemens was the first to introduce a fully integrated whole-body integrated scanner, called Biograph mMR, in 2010 (Figure 1, left). The MR component of the Biograph mMR consists 
of a $60-\mathrm{cm}$ bore 3-T niobium-titanium superconductor magnet, an actively shielded wholebody gradient coil system ( $45 \mathrm{mT} / \mathrm{m}$ amplitude and $200 \mathrm{~T} / \mathrm{m} / \mathrm{s}$ slew rate) and a radiofrequency body coil. Similar to the BrainPET prototype, the PET component of the mMR also uses APD technology. However, instead of placing the PET detectors inside the MR bore, they are positioned between the body radiofrequency coil and the gradient set. Eight rings of 56 detectors blocks make up the whole PET gantry. Each detector block consists of an $8 \times 8$ array of $4 \times 4 \times 20 \mathrm{~mm}^{3}$ LSO crystals readout by a $3 \times 3$ array of Hamamatsu APDs. The transaxial/axial fields of view are $59.4 / 25.8 \mathrm{~cm}$. To address the need for compensating for temperature changes during the MR acquisition, chilled water is used to control the temperature inside the detector modules. In terms of performance, the reported energy resolution is $14.5 \%$, the timing resolution is $2.93 \mathrm{~ns}$, the spatial resolution is $4.3 \mathrm{~mm}$ FWHM at $1 \mathrm{~cm}$ offset from the center of the field of view and the scanner sensitivity is 15.0 $\mathrm{kcps} / \mathrm{MBq}$ (43). The mMR scanner can acquire list mode data in 3D mode. The PET images can be reconstructed using either the filtered backprojection or the 3D ordinary Poisson ordered-subset expectation maximization algorithm.

\section{GENERAL ELECTRIC SIGNA PET/MRI}

General Electric introduced the first whole-body SiPM-based integrated PET-MRI scanner, called SIGNA PET/MR, in 2013 (Figure 1, right). The MR component of the Signa PET/MR scanner consists of a $60-\mathrm{cm}$ bore 3-T superconductive magnet, gradient coils (44 $\mathrm{mT} / \mathrm{m}$ amplitude and $200 \mathrm{~T} / \mathrm{m} / \mathrm{s}$ slew rate) and transmit/receive body coils similar to the original 3T MR 750w MR scanner on which it is based. The PET components are placed between the radiofrequency shield of the body coil and the gradient coils. The PET gantry is made up of 28 modules consisting of 20 detector blocks made up of a $4 \times 9$ array of $4.0 \times$ $5.3 \times 25 \mathrm{~mm}^{3}$ lutetium based scintillator (LBS) readout by $1 \times 3$ arrays of Hamamatsu SiPMs. The transaxial/axial fields of view are $62 / 25 \mathrm{~cm}$. Chilled water is also used to control the temperature based on the feedback received from 280 thermistors positioned throughout the scanner. In terms of performance, the reported energy resolution is $10.3 \%$, the timing resolution is less than $400 \mathrm{ps}$, the spatial resolution is $4.4 \mathrm{~mm}$ FWHM at $1 \mathrm{~cm}$ offset from the center of the field of view and the scanner sensitivity is $23.3 \mathrm{kcps} / \mathrm{MBq}(44)$.

\section{MR-based PET attenuation correction}

The need for deriving the attenuation maps from the MR data was a significant methodological hurdle that human PET/MRI had to face from the very beginning given the very different physical principles that underlie the signal generation in the two modalities. Specifically, the MR signal depends on proton density and tissue relaxation times and it does not reflect electron density, which is relevant for attenuation correction. In this section, we introduce the standard protocols for generating the attenuation maps available on the Biograph mMR and Signa PET/MRI scanners, discuss specific aspects that need to be considered in an integrated PET/MRI scanner (some of the solutions that have been proposed for addressing these issues are discussed in detail in Yasheng Chen and Hongyu An's article, "Attenuation Correction of PET/MR Imaging," in this issue, and comment on the potential benefits of using the TOF information for improving the accuracy of MR-based attenuation correction methods. 


\section{STANDARD PROTOCOLS APPROVED FOR CLINICAL USE}

On the Biograph mMR, the attenuation correction is performed using the MR data acquired with a 2-point Dixon volume interpolated breath-hold exam (VIBE) MR sequence. In-phase, out-of-phase, water and fat images are generated at each bed position and combined to generate the corresponding whole-body images (Figure 2, left). The in-phase images are first thresholded to separate the voxels corresponding to the subject from the background air. An atlas-based approach is next used to segment the lungs. The voxels corresponding to fat and soft tissue voxels are obtained from the fat and water images. Additional post processing is performed to remove the skin voxels and noise. Finally, four tissue classes (background air, lung, fat and soft tissue) are segmented and predefined linear attenuation coefficients ( 0 , $0.022,0.085,0.100 \mathrm{~cm}^{-1}$, respectively) are assigned to generate the attenuation map (Figure 2 , right). Bone tissue is misclassified as soft tissue (45).

Similarly, on the Signa PET/MRI scanner, the attenuation information is acquired using a LAVA-Flex scan (46) and the resulting Dixon images are segmented into air, lung, fat and water. Bone tissue is again misclassified as soft tissue with the exception of the head where an atlas-based registration is used to account for the skull attenuation (47).

\section{SPECIAL CONSIDERATIONS}

Although MR-based attenuation correction eliminates the radiation exposure associated with the CT exam in PET/CT scanners (of interest in at-risk patient populations), several practical issues limit the accuracy of the currently available methods.

Bone imaging-Imaging bone tissue is extremely difficult using standard MR sequences and thus the corresponding voxels cannot be easily identified to be included in the attenuation map. However, bone tissue has the highest linear attenuation coefficients and cannot be ignored without biasing the PET measurements.

Truncation artifacts-Because of the long duration of a comprehensive whole-body PET/MRI exam, the patients are scanned with their arms in the field of view. In MRI, geometrical distortions are present in the case of large fields of view (i.e. $>\sim 45 \mathrm{~cm}$ diameter), which results in complete or partial truncation of the arms or other body regions and, consequently, of the attenuation maps derived from these MR images. As a result, bias in PET quantification could be introduced (48), potentially compromising the clinical interpretability of the PET images. On the Biograph mMR scanner, a maximum likelihood activity and attenuation (MLAA) estimation algorithm was implemented to recover the missing data $(49,50)$. At each iteration, first the activity is updated maintaining the linear attenuation coefficients constant and second the attenuation map is updated while keeping the emission image constant. When successful, this method was shown to reduce the error in the SUV estimation from 15-50\% to less than 5\% (51). A similar algorithm that uses the non-attenuation corrected TOF reconstructed PET image to estimate the patient outline is available on the Signa PET/MRI scanner $(44,52)$.

Metallic implants-Foreign objects present in the body (e.g. prosthetic devices, dental implants, subcutaneous chemotherapy ports and surgical clips) can introduce susceptibility 
artifacts in the MR images, leading to signal voids in or distortions of the MR images (5355) that propagate in the attenuation maps derived from these data. Novel MR sequences have been proposed to minimize the susceptibility artifacts in the MR images in the presence of metallic implants (56-59) and they could minimize this source of bias.

Lung imaging-Imaging the lung parenchyma using conventional MR sequences is extremely challenging due to its very short $\mathrm{T}_{2} *$ (i.e. $0.74 \mathrm{~ms}$ at $3 \mathrm{~T}(60)$ ), low proton density and subject motion. However, there is large variability in lung attenuation $(45,61)$, not only when comparing diseased versus normal tissue but also in the different phases of the respiratory cycle (62).

MR hardware-Although the radiofrequency coils have to be redesigned and built using minimally attenuating materials, not accounting for their remaining attenuation in the correction procedure could still bias the PET measurements. The attenuation templates for several of the standard coils (e.g. head and neck coil, spine) are provided by the manufacturers while the flexible body coils are ignored in the attenuation correction procedure.

\section{THE POTENTIAL ADDED VALUE OF THE TOF INFORMATION}

The major difference between the two integrated PET/MRI scanners that are currently commercially available is that the Signa PET/MR scanner has TOF capability. The main advantage of incorporating the TOF information is that the signal-to-noise ratio (SNR) improves compared to the non-TOF images. The various ways in which this can benefit clinical PET studies (e.g. improved image quality and lesion detection, shorten the scan time or reduce the administered dose) have been discussed extensively in the context of PET/CT (63-68).

Although both vendors seem to agree on the value of TOF in the case of integrated PET/CT scanners, there is still a debate on this topic when it comes to PET/MRI. In the context of MR-based attenuation correction, the TOF information might prove useful for mitigating some of the remaining challenges and artifacts described above.

The standard MR-based attenuation correction methods as well as the majority of the more advanced ones presented in Yasheng Chen and Hongyu An's article, "Attenuation Correction of PET/MR Imaging," in this issue, share the same limitation - using only the MR data and templates of linear attenuation coefficients it is impossible to account for large intersubject variability. One approach to overcome this limitation is to incorporate the information inherently present in the PET emission data (69-73). This is because the anatomical information provided by the MR or the TOF minimizes the attenuation-emission estimation "cross-talk" that has limited the usefulness of the early approaches (i.e. any errors in the estimation of emission image lead to compensatory errors in the estimation of the attenuation image). For example, a method similar to the MLAA discussed above for addressing the arms truncation problem and the TOF information were used to improve the estimation of the linear attenuation coefficients assigned to the different regions segmented from the MR data (72). 
Several recent studies have shown the TOF can reduce the effect of inconsistencies between the emission and attenuation data, such as those encountered in MR-based attenuation correction due to bone or lung tissue misclassification, incomplete attenuation maps caused by truncation or implant related signal voids.

For example, Mehranian and Zaidi used CT data to generate 4-class segmented attenuation maps that were used to reconstruct the PET data with and without using the TOF information. They noted significant changes between the two methods in vertebra, pelvis and cerebrum but not in the lungs, aorta and liver (Figure 3). The TOF reconstruction was also able to reduce the artifacts related to metallic implants and those introduced by respiratory motion (4). Although TOF could theoretically reduce the effect of some of these artifacts, the actual impact is still limited given the TOF performance of current generation PET/MRI scanners (4).

Delso et al. studied the impact of TOF on the artifacts related to respiratory mismatch between the emission and attenuation data by comparing the standardized uptake values (SUVs) measured in FDG-avid lesions between the different reconstructions. The range of the differences in maximum SUVs decreased from $-18 \%$ to $26 \%$ for the non-TOF to $-15 \%$ to $18 \%$ for the TOF reconstruction. However, the authors also reported that the smaller magnitude errors observed in the TOF reconstruction spread over larger areas compared to the non-TOF reconstruction (74). Similarly, Davison et al. estimated the mean error across the signal void introduced by a hip implant is reduced by $40 \%$ when using TOF reconstruction. However, they also reported more (smaller magnitude) errors away from the artifact and ignored the implant attenuation in their simulations (75).

Minamimoto et al. studied the impact of TOF on the image quality, focusing on the artifacts related to dental implants, breathing-induced mismatches and inaccurate scatter correction due to high bladder FDG concentration. Two board-certified nuclear medicine physicians assessed the quality of the PET images on a 3-point scale. Although a trend for improved image quality using the TOF information was observed, the only difference between the visual assessment scores that reached statistical significance was reported in the case of the pelvic artifacts (76).

At least initially, favoring a tested and more mature photon detector - APD - over the novel TOF-capable one - SiPM - seemed justified by the desire to first focus on exploring the potential research and clinical applications of PET/MRI. However, as the gap in the level of maturity between the two detector technologies diminishes and especially if the timing resolution of SiPM-based detectors improves, it is likely that both vendors (and perhaps some of the other major medical imaging equipment manufacturers) will start using SiPMs for their future PET/MRI scanners. In fact, the recent introduction by GE of a SiPM-based PET/CT scanner suggests that SiPMs could become the technology of choice even in $\mathrm{PET} / \mathrm{CT}$ at least for the foreseeable future until another even higher performance detector technology emerges. 


\section{Promising research and clinical applications}

In the following articles, several methodological developments as well as proof-of-principle studies and promising applications that could benefit from the simultaneous acquisition of complementary data are presented. Here we only briefly introduce some of the potential research and clinical applications to discuss opportunities opened up by the simultaneous acquisition.

\section{OPPORTUNITIES FOR METHODOLOGICAL CROSS-VALIDATION AND IMPROVEMENT}

After an in depth discussion of the advanced methods proposed for deriving more accurate attenuation maps from the MR data, in particular for neurological applications, is given in Yasheng Chen and Hongyu An's article, “Attenuation Correction of PET/MR Imaging," in this issue, approaches to improve the PET images by performing MR-assisted motion correction (as well as by incorporating the MR-derived higher resolution morphological information (25)) are discussed in David S. Lalush's article, "MR-derived Improvements in PET Imaging," in this issue. MR can be used for deriving high temporal resolution estimates to characterize both rigid and non-rigid body as well as bulk motion (77). The reduction of motion-related artifacts and blurring could have an immediate positive impact on clinical studies while the improved quantification could benefit more advanced applications. Although PET data-driven approaches for characterizing the respiratory (78) and cardiac (79-84) motion are not discussed in detail in Yasheng Chen and Hongyu An's article, "Attenuation Correction of PET/MR Imaging," in this issue, developing such methods has been a very active area of research in PET/CT for many years (85). This was required because $\mathrm{CT}$ could not be used motion characterization due to the sequential nature of the acquisitions and the increased radiation exposure involved in repeated CT examinations. Since these are not issues in integrated PET/MRI scanners, the various techniques that have been proposed for MR- and PET-assisted motion characterization could finally be compared and improved. The information derived from both modalities could even be used to jointly estimate the motion (86).

The simultaneous acquisition would also allow the cross-validation of different techniques that have been proposed to measure various biological processes. As an example, crossvalidating cerebral perfusion measurements could benefit stroke patients, one of the potential neurological applications discussed in Michelle M. Miller-Thomas and Tammie L.S. Benzinger's article, "Neurological Applications of PET/MR," in this issue. $\mathrm{H}_{2}{ }^{15} \mathrm{O}$ PET is considered the "gold standard" for measuring cerebral blood flow, but several MR methods are also available for the same purpose $(87,88)$. Perhaps not surprisingly, there are notable discrepancies between PET and MRI cerebral blood flow measures performed separately (89) and it is not clear if this is due to true technique variations or caused by actual differences in the physiological state between distinct measurement periods. Although PET/MRI examinations are very difficult to routinely perform in the acute phase of stroke, this much needed cross-validation could help elucidate the ongoing debate concerning the relationship between the perfusion-diffusion mismatch and the PET penumbra (90-93), which would have immediate clinical implications. The first such study performed on an integrated PET/MRI device revealed discrepancies between the PET- and MRI-derived 
perfusion measurements that the authors attributed to the limited accuracy of current MRI based techniques (94).

A similar case could be made for the need and opportunity to cross-validate various PET and MR methods of relevance to cardiac applications (please see Jeffrey M.C. Lau, Richard Laforest, Felix Nensa, et al.'s article, "Cardiac Applications of PET/MR Imaging," in this issue). For example, FDG PET and late gadolinium enhancement MRI both provide extremely accurate assessments of myocardial viability via highly different mechanisms. While qualitative myocardial perfusion is being increasingly used clinically, quantitative flow measurement by MRI is still challenging and would benefit from the validation provided by a simultaneous PET measurement. Since perfusion studies involve a pharmacological challenge and a highly dynamic signal, simultaneous acquisition of the PET and MRI data is needed to allow a true comparison of the techniques to be performed under identical conditions (95).

\section{OPPORTUNITIES FOR SYNERGISTIC USE OF THE MULTIMODAL INFORMATION}

Neurology/psychiatry/neuroscience-PET and MRI provide complementary information in the assessment of many neurological disorders (please see Michelle M. Miller-Thomas and Tammie L.S. Benzinger's article, "Neurological Applications of PET/ MR," in this issue). The improvement in PET data quantification using the MR-assisted methods discussed in David S. Lalush's article, "MR-derived Improvements in PET Imaging," in this issue, could benefit virtually all these applications. An example of incorporating anatomical information derived from MR in the PET image reconstruction is shown in Figure 4.

In Alzheimer's disease, PET is used to characterize the amyloid and tau buildup while MR can inform about neuronal degeneration and cortical dysfunction (96-100) and acquiring these data in a single imaging session would increase patient convenience. Additionally, motion and partial volume effects correction are particularly relevant in this patient population to address two of the most important confounding factors that bias the interpretation of PET measurements from small and anatomically complex structures (e.g. hippocampus).

In addition to evaluating changes in metabolism and anatomy in the study of normal and diseased brain, PET/MRI allows the simultaneous assessment of brain neurochemistry and activity. For example, the interaction between dopamine signaling and neural networks changes during working memory was investigated in a recent study (28). Studying the temporal correlation between these biomarkers is an even more exciting opportunity that will likely contribute to improving our understanding of many neuropsychiatric diseases. In the first study of this type, the temporospatial relationship between changes in dopamine receptor occupancy (measured with PET) and cerebral blood volume (measured with fMRI) in response to the administration of pharmacological doses of a D2/D3 receptor antagonist was assessed (29). Subsequently, the temporal dissociation between the signals was assessed using D2/D3 agonists and antagonists and the results suggest that simultaneous PET/fMRI may enable for the first time the in vivo characterization of receptor desensitization and internalization (Figure 5) (1). 
Oncology-In the assessment of oncological patients, the synergistic use of imaging biomarkers reflecting changes in anatomy (from MRI) and metabolism (from PET) has the potential to increase confidence in equivocal cases both at the initial staging and during follow-up (101). Most of the early PET/MRI adopters have first focused on comparing its performance to that of PET/CT for various oncological applications and the preliminary results suggest there are certain indications for which PET/MRI might be superior the PET/CT. Perhaps the most obvious benefit of obtaining information about the anatomy with MRI instead of CT is when scanning patients for whom radiation exposure is of concerns such as pediatric patients, although additional issues unique to pediatric imaging need to be considered (please see Yueh Z. Lee and Franz Wolfgang Hirsch's article, "Pediatric Applications of Hybrid PET / MRI, in this issue). PET/MRI will likely be the modality of choice in areas of the body where $\mathrm{CT}$ is suboptimal for providing anatomical correlates to the PET metabolic measurements because of the poor soft tissue contrast such as head and neck (please see Yueh Z. Lee, Joana Ramalho and Brice Kessler's article, "PET MR in Head and Neck Imaging," in this issue) and the male and female pelvis (please see Jorge D. Oldan, Shetal N. Shah and Tracy Lynn Rose's article, "Applications of PET/MR Imaging in Uro-Gynecologic and Genitourinary Cancers," in this issue). PET/MRI will also be preferred in areas in which MR imaging provides improved tissue specificity such as liver, breast and bone marrow (e.g. see Shetal N. Shah and Jorge D. Oldan's article, "PET/MR Imaging of Multiple Myeloma," in this issue for a more in depth discussion of the current use and potential applications of PET/MRI in multiple myeloma).

MR-based non-rigid body motion correction techniques (please see David S. Lalush's article, "MR-derived Improvements in PET Imaging," in this issue) can reduce blurring and improve the detection of small lesions. An example of how this unique capability of simultaneous PET/MR could improve the detection of pulmonary nodules is given in Fernando E. Boada, Thomas Koesters, Kai Tobias Block and Hersh Chandarana's article, "Improved Detection of Small Pulmonary Nodules Through Simultaneous MR/PET Imaging," in this issue.

In glioblastoma, MRI allows the assessment of microvascular proliferation and permeability while PET can inform about tumor metabolism, proliferation and even the delivery of radiolabeled chemotherapeutic agents (102). Using mathematical models to combine the multimodal quantitative information obtained in an integrated PET/MRI scanner could help us better understand the tumor biology and the mechanisms of action of promising therapeutic agents (103). The potential uses of multimodal PET/MRI data in radiation therapy for improved target delineation as well as for the evaluation of tumor response are discussed in Tong Zhu, Shiva Das and Terence Wong's article, "Integration of PET/MR Hybrid Imaging into Radiation Therapy Treatment," in this issue.

Cardiology-PET and MRI are widely used in the clinical assessment of patients with cardiovascular disease, providing complementary information about molecular and metabolic processes (PET) and anatomy and function (MRI). The technical challenges specific to cardiac imaging and the potential clinical applications (e.g. myocardial viability and perfusion, carotid atherosclerotic plague, etc.) that could benefit from the simultaneous acquisition are discussed in Jeffrey M.C. Lau, Richard Laforest, Felix Nensa, et al.'s article, 
"Cardiac Applications of PET/MR Imaging," in this issue. In this context, the non-rigid body MR-assisted motion correction methods described in David S. Lalush's article, "MRderived Improvements in PET Imaging," in this issue, have again the potential to significantly improve PET data quantification and reproducibility.

\section{References}

1. Sander CY, Hooker JM, Catana C, et al. Imaging Agonist-Induced D2/D3 Receptor Desensitization and Internalization In Vivo with PET/fMRI. Neuropsychopharmacology. 2016; 41:1427-1436. [PubMed: 26388148]

2. Siemens. [Accessed January 4, 2017] https://usa.healthcare.siemens.com/magnetic-resonanceimaging/mr-petscanner/biograph-mmr.

3. GE. [Accessed January 4, 2017] http://www3.gehealthcare.com/en/products/categories/ magnetic_resonance_imaging/3-0t/signa_pet-mr.

4. Mehranian A, Zaidi H. Impact of Time-of-Flight PET on Quantification Errors in MR ImagingBased Attenuation Correction. J Nucl Med. 2015; 56:635-641. [PubMed: 25745090]

5. Townsend DW, Carney JP, Yap JT, et al. PET/CT today and tomorrow. J Nucl Med. 2004; 45(Suppl 1):4S-14S. [PubMed: 14736831]

6. Shao Y, Cherry SR, Farahani K, et al. Simultaneous PET and MR imaging. Phys Med Biol. 1997; 42:1965-1970. [PubMed: 9364592]

7. Mackewn JE, Strul D, Hallett WA, et al. Design and development of an MR-compatible PET scanner for imaging small animals. IEEE Trans Nucl Sci. 2005; 52:1376-1380.

8. Mackewn JE, Halsted P, Charles-Edwards G, et al. Performance Evaluation of an MRI-Compatible Pre-Clinical PET System Using Long Optical Fibers. IEEE Trans Nucl Sci. 2010; 57:1052-1062.

9. Raylman RR, Majewski S, Lemieux SK, et al. Simultaneous MRI and PET imaging of a rat brain. Phys Med Biol. 2006; 51:6371-6379. [PubMed: 17148823]

10. Raylman RR, Majewski S, Velan SS, et al. Simultaneous acquisition of magnetic resonance spectroscopy (MRS) data and positron emission tomography (PET) images with a prototype MRcompatible, small animal PET imager. J Magn Reson. 2007; 186:305-310. [PubMed: 17433742]

11. Yamamoto S, Imaizumi M, Kanai Y, et al. Design and performance from an integrated PET/MRI system for small animals. Ann Nucl Med. 2010; 24:89-98. [PubMed: 20058103]

12. Tatsumi M, Yamamoto S, Imaizumi M, et al. Simultaneous PET/MR body imaging in rats: initial experiences with an integrated PET/MRI scanner. Ann Nucl Med. 2012; 26:444-449. [PubMed: 22359223]

13. Lucas AJ, Hawkes RC, Ansorge RE, et al. Development of a combined microPET((R))-MR system. Technol Cancer Res Treat. 2006; 5:337-341. [PubMed: 16866564]

14. Bindseil GA, Gilbert KM, Scholl TJ, et al. First Image From a Combined Positron Emission Tomography and Field-Cycled MRI System. Magn Reson Med. 2011; 66:301-305. [PubMed: 21695730]

15. Peng H, Handler WB, Scholl TJ, et al. Proof-of-principle study of a small animal PET/field-cycled MRI combined system using conventional PMT technology. Nucl Instrum Methods Phys Res Sect A-Accel Spectrom Dect Assoc Equip. 2010; 612:412-420.

16. Pichler B, Lorenz E, Mirzoyan R, et al. Performance test of a LSO-APD PET module in a 9.4 Tesla magnet. Paper presented at: Nuclear Science Symposium, 1997. IEEE. 1997

17. Catana C, Procissi D, Wu Y, et al. Simultaneous in vivo positron emission tomography and magnetic resonance imaging. Proc Natl Acad Sci USA. 2008; 105:3705-3710. [PubMed: 18319342]

18. Judenhofer MS, Wehrl HF, Newport DF, et al. Simultaneous PET-MRI: a new approach for functional and morphological imaging. Nat Med. 2008; 14:459-465. [PubMed: 18376410]

19. Maramraju SH, Smith SD, Junnarkar SS, et al. Small animal simultaneous PET/MRI: initial experiences in a 9.4 T microMRI. Phys Med Biol. 2011; 56:2459-2480. [PubMed: 21441651]

Magn Reson Imaging Clin N Am. Author manuscript; available in PMC 2018 May 01. 
20. Schlemmer H-PW, Pichler BJ, Schmand M, et al. Simultaneous MR/PET imaging of the human brain: feasibility study. Radiology. 2008; 248:1028-1035. [PubMed: 18710991]

21. Kolb A, Wehrl HF, Hofmann M, et al. Technical performance evaluation of a human brain PET/MRI system. Eur Radiol. 2012; 22:1776-1788. [PubMed: 22752524]

22. Chonde DB, Abolmaali N, Arabasz G, et al. Effect of MRI Acoustic Noise on Cerebral Fludeoxyglucose Uptake in Simultaneous MR-PET Imaging. Invest Radiol. 2013; 48:302-312. [PubMed: 23462677]

23. Catana C, Benner T, van der Kouwe A, et al. MRI-assisted PET motion correction for neurologic studies in an integrated MR-PET scanner. J Nucl Med. 2011; 52:154-161. [PubMed: 21189415]

24. Catana C, van der Kouwe A, Benner T, et al. Toward implementing an MRI-based PET attenuation-correction method for neurologic studies on the MR-PET brain prototype. J Nucl Med. 2010; 51:1431-1438. [PubMed: 20810759]

25. Hutchcroft W, Wang G, Chen KT, et al. Anatomically-aided PET reconstruction using the kernel method. Phys Med Biol. 2016; 61:6668-6683. [PubMed: 27541810]

26. Izquierdo-Garcia D, Hansen AE, Forster S, et al. An SPM8-based approach for attenuation correction combining segmentation and nonrigid template formation: application to simultaneous PET/MR brain imaging. J Nucl Med. 2014; 55:1825-1830. [PubMed: 25278515]

27. Catana C, Drzezga A, Heiss WD, et al. PET/MRI for Neurologic Applications. J Nucl Med. 2012; 53:1916-1925. [PubMed: 23143086]

28. Roffman JL, Tanner AS, Eryilmaz H, et al. Dopamine D-1 signaling organizes network dynamics underlying working memory. Science advances. 2016; 2:10.

29. Sander CY, Hooker JM, Catana C, et al. Neurovascular coupling to D2/D3 dopamine receptor occupancy using simultaneous PET/functional MRI. Proc Natl Acad Sci USA. 2013; 30:30.

30. Wey HY, Catana C, Hooker JM, et al. Simultaneous fMRI-PET of the opioidergic pain system in human brain. Neuroimage. 2014; 102(Pt 2):275-282. [PubMed: 25107855]

31. Loggia ML, Chonde DB, Akeju O, et al. Evidence for brain glial activation in chronic pain patients. Brain. 2015; 138:604-615. [PubMed: 25582579]

32. Zurcher NR, Loggia ML, Lawson R, et al. Increased in vivo glial activation in patients with amyotrophic lateral sclerosis: assessed with [(11)C]-PBR28. NeuroImage Clinical. 2015; 7:409_ 414. [PubMed: 25685708]

33. Catana C, Guimaraes AR, Rosen BR. PET and MR Imaging: The Odd Couple or a Match Made in Heaven? J Nucl Med. 2013; 54:815-824. [PubMed: 23492887]

34. Frullano L, Catana C, Benner T, et al. Bimodal MR-PET Agent for Quantitative pH Imaging. Angew Chem-Int Edit. 2010; 49:2382-2384.

35. Uppal R, Catana C, Ay I, et al. Simultaneous MR-PET imaging of thrombus with a fibrin-targeted dual MR-PET probe: a feasibility study. Radiology. 2011; 258:812-820. [PubMed: 21177389]

36. Seong Jong $\mathrm{H}$, In Chan S, Ito M, et al. An investigation into the use of Geiger-mode solid-state photomultipliers for simultaneous PET and MRI acquisition. IEEE Trans Nucl Sc. 2008; 55:882888.

37. Yamamoto S, Watabe H, Kanai Y, et al. Interference between PET and MRI sub-systems in a silicon-photomultiplier-based PET/MRI system. Phys Med Biol. 2011; 56:4147-4159. [PubMed: 21693791]

38. Yamamoto S, Watabe T, Watabe H, et al. Simultaneous imaging using Si-PM-based PET and MRI for development of an integrated PET/MRI system. Phys Med Biol. 2012; 57:N1. [PubMed: 22170810]

39. Yoon HS, Ko GB, Il Kwon S, et al. Initial Results of Simultaneous PET/MRI Experiments with an MRI-Compatible Silicon Photomultiplier PET Scanner. J Nucl Med. 2012; 53:608-614. [PubMed: 22414638]

40. Kang J, Choi Y, Hong KJ, et al. A small animal PET based on GAPDs and charge signal transmission approach for hybrid PET-MR imaging. J Instrum. 2011; 6

41. Hong SJ, Kang HG, Ko GB, et al. SiPM-PET with a short optical fiber bundle for simultaneous PET-MR imaging. Phys Med Biol. 2012; 57:3869-3883. [PubMed: 22644119] 
42. Yamamoto S, Kuroda K, Senda M. Scintillator selection for MR-compatible gamma detectors. IEEE Trans Nucl Sc. 2003; 50:1683-1685.

43. Delso G, Furst S, Jakoby B, et al. Performance measurements of the Siemens mMR integrated whole-body PET/MR scanner. J Nucl Med. 2011; 52:1914-1922. [PubMed: 22080447]

44. Levin CS, Maramraju H, Khalighi MM, et al. Design Features and Mutual Compatibility Studies of the Time-of-Flight PET Capable GE SIGNA PET/MR System. IEEE TMI. 2016; 35:1907-1914.

45. Martinez-Moller A, Souvatzoglou M, Delso G, et al. Tissue classification as a potential approach for attenuation correction in whole-body PET/MRI: evaluation with PET/CT data. J Nucl Med. 2009; 50:520-526. [PubMed: 19289430]

46. Wollenweber SD, Ambwani S, Lonn AHR, et al. Comparison of 4-Class and Continuous Fat/Water Methods for Whole-Body, MR-Based PET Attenuation Correction. IEEE Trans Nucl Sci. 2013; 60:3391-3398.

47. Wollenweber SD, Ambwani S, Delso G, et al. Evaluation of an Atlas-Based PET Head Attenuation Correction Using PET/CT \& MR Patient Data. IEEE Trans Nucl Sci. 2013; 60:3383-3390.

48. Delso G, Martinez-Moller A, Bundschuh RA, et al. The effect of limited MR field of view in MR/PET attenuation correction. Med Phys. 2010; 37:2804-2812. [PubMed: 20632591]

49. Nuyts J, Dupont P, Stroobants S, et al. Simultaneous maximum a posteriori reconstruction of attenuation and activity distributions from emission sinograms. IEEE TMI. 1999; 18:393-403.

50. Nuyts J, Bal G, Kehren F, et al. Completion of a Truncated Attenuation Image From the Attenuated PET Emission Data. Medical Imaging, IEEE Transactions on. 2013; 32:237-246.

51. Nuyts, J., Michel, C., Fenchel, M., et al. 2010 Ieee Nuclear Science Symposium Conference Record. New York: Ieee; 2010. Completion of a Truncated Attenuation Image from the Attenuated PET Emission Data; p. 2123-2127.

52. Qian, R., Manjeshwar, RM., Ambwani, S., et al. Truncation Completion of MR-based PET Attenuation Maps Using Time-of-flight Non-attenuation-corrected PET Images. In: Yu, B., editor. 2012 Ieee Nuclear Science Symposium and Medical Imaging Conference Record. New York: Ieee; 2012. p. 2773-2775.

53. Hargreaves BA, Worters PW, Pauly KB, et al. Metal-induced artifacts in MRI. Am J Roentgenol. 2011; 197:547-555. [PubMed: 21862795]

54. Genson CC, Blane CE, Helvie MA, et al. Effects on breast MRI of artifacts caused by metallic tissue marker clips. Am J Roentgenol. 2007; 188:372-376. [PubMed: 17242244]

55. Le Y, Kipfer HD, Majidi SS, et al. Comparison of the artifacts caused by metallic implants in breast MRI using dual-echo dixon versus conventional fat-suppression techniques. Am J Roentgenol. 2014; 203:W307-W314. [PubMed: 25148189]

56. Ai T, Padua A, Goerner F, et al. SEMAC-VAT and MSVAT-SPACE Sequence Strategies for Metal Artifact Reduction in 1.5T Magnetic Resonance Imaging. Invest Radiol. 2012; 47:267-276. [PubMed: 22266987]

57. Sutter R, Ulbrich EJ, Jellus V, et al. Reduction of Metal Artifacts in Patients with Total Hip Arthroplasty with Slice-encoding Metal Artifact Correction and View-Angle Tilting MR Imaging. Radiology. 2012; 265:204-214. [PubMed: 22923720]

58. Zho SY, Kim MO, Lee KW, et al. Artifact reduction from metallic dental materials in T1-weighted spin-echo imaging at 3.0 tesla. J Magn Reson Imaging. 2013; 37:471-478. [PubMed: 22941956]

59. Choi SJ, Koch KM, Hargreaves BA, et al. Metal artifact reduction with MAVRIC SL at 3-T MRI in patients with hip arthroplasty. Am J Roentgenol. 2015; 204:140-147. [PubMed: 25539249]

60. Yu J, Xue Y, Song HK. Comparison of lung T2* during free-breathing at $1.5 \mathrm{~T}$ and $3.0 \mathrm{~T}$ with ultrashort echo time imaging. Magn Reson Med. 2011; 66:248-254. [PubMed: 21695727]

61. Schulz V, Torres-Espallardo I, Renisch S, et al. Automatic, three-segment, MR-based attenuation correction for whole-body PET/MR data. Eur J Nucl Med Mol Imaging. 2011; 38:138-152. [PubMed: 20922522]

62. Marshall HR, Prato FS, Deans L, et al. Variable lung density consideration in attenuation correction of whole-body PET/MRI. J Nucl Med. 2012

63. Conti M. Focus on time-of-flight PET: the benefits of improved time resolution. Eur J Nucl Med Mol Imaging. 2011; 38:1147-1157. [PubMed: 21229244] 
64. Surti S, Karp JS. Advances in time-of-flight PET. Phys Medica. 2016; 32:12-22.

65. Vandenberghe S, Mikhaylova E, D'Hoe E, et al. Recent developments in time-of-flight PET. Ejnmmi Physics. 2016; 3:30. [PubMed: 27957730]

66. Eriksson L, Conti M. Randoms and TOF gain revisited. Phys Med Biol. 2015; 60:1613-1623. [PubMed: 25615713]

67. Karp JS, Surti S, Daube-Witherspoon ME, et al. Benefit of time-of-flight in PET: Experimental and clinical results. J Nucl Med. 2008; 49:462-470. [PubMed: 18287269]

68. Westerwoudt V, Conti M, Eriksson L. Advantages of Improved Time Resolution for TOF PET at Very Low Statistics. IEEE Trans Nucl Sci. 2014; 61:126-133.

69. Mollet P, Keereman V, Clementel E, et al. Simultaneous MR-Compatible Emission and Transmission Imaging for PET Using Time-of-Flight Information. Medical Imaging, IEEE Transactions on. 2012; 31:1734-1742.

70. Panin VY, Aykac M, Casey ME. Simultaneous reconstruction of emission activity and attenuation coefficient distribution from TOF data, acquired with external transmission source. Phys Med Biol. 2013; 58:3649-3669. [PubMed: 23648397]

71. Rezaei A, Defrise M, Bal G, et al. Simultaneous Reconstruction of Activity and Attenuation in Time-of-Flight PET. Medical Imaging, IEEE Transactions on. 2012; 31:2224-2233.

72. Salomon A, Goedicke A, Schweizer B, et al. Simultaneous reconstruction of activity and attenuation for PET/MR. IEEE Trans Med Imaging. 2011; 30:804-813. Epub 2092010 Nov 2095429. [PubMed: 21118768]

73. Censor Y, Gustafson DE, Lent A, et al. A New Approach to the Emission Computerized Tomography Problem: Simultaneous Calculation of Attenuation and Activity Coefficients. IEEE Trans Nucl Sc. 1979; 26:2775-2779.

74. Gaspar D, Mohammed K, Edwin ter V, et al. Effect of Time-of-Flight Information on PET/MR Reconstruction Artifacts: Comparison of Free-breathing versus Breath-hold MR-based Attenuation Correction. Radiology. 2016; 0:152509.

75. Davison H, ter Voert EE, de Galiza Barbosa F, et al. Incorporation of Time-of-Flight Information Reduces Metal Artifacts in Simultaneous Positron Emission Tomography/Magnetic Resonance Imaging: A Simulation Study. Invest Radiol. 2015; 50:423-429. [PubMed: 25756682]

76. Minamimoto R, Levin C, Jamali M, et al. Improvements in PET Image Quality in Time of Flight (TOF) Simultaneous PET/MRI. Mol Imaging Biol. 2016; 18:776-781. [PubMed: 26884058]

77. Catana C. Motion correction options in PET/MRI. Semin Nucl Med. 2015; 45:212-223. [PubMed: 25841276]

78. Dawood M, Buther F, Jiang X, et al. Respiratory motion correction in 3-D PET data with advanced optical flow algorithms. IEEE Trans Med Imaging. 2008; 27:1164-1175. [PubMed: 18672433]

79. Gilland DR, Mair BA, Parker JG. Motion estimation for cardiac emission tomography by optical flow methods. Phys Med Biol. 2008; 53:2991-3006. [PubMed: 18475004]

80. Klein GJ, Reutter BW, Huesman RH. Non-rigid summing of gated PET via optical flow. IEEE Trans Nucl Sci. 1997; 44:1509-1512.

81. Dawood M, Gigengack F, Jiang XY, et al. A mass conservation-based optical flow method for cardiac motion correction in 3D-PET. Med Phys. 2013; 40:9.

82. Gigengack F, Ruthotto L, Burger M, et al. Motion correction in dual gated cardiac PET using masspreserving image registration. IEEE Trans Med Imaging. 2012; 31:698-712. [PubMed: 22084048]

83. Bai W, Brady M. Regularized B-spline deformable registration for respiratory motion correction in PET images. Phys Med Biol. 2009; 54:2719-2736. [PubMed: 19351979]

84. Lamare F, Ledesma Carbayo MJ, Cresson T, et al. List-mode-based reconstruction for respiratory motion correction in PET using non-rigid body transformations. Phys Med Biol. 2007; 52:51875204. [PubMed: 17762080]

85. Kesner AL, Schleyer PJ, Büther F, et al. On transcending the impasse of respiratory motion correction applications in routine clinical imaging-a consideration of a fully automated data driven motion control framework. EJNMMI Physics. 2014; 1:8. [PubMed: 26501450]

86. Fieseler M, Gigengack F, Jiang X, et al. Motion correction of whole-body PET data with a joint PET-MRI registration functional. Biomed Eng Online. 2014; 13(Suppl 1):S2. 
87. Villringer A, Rosen BR, Belliveau JW, et al. Dynamic imaging with Lanthanide chelates in normal brain - contrast due to magnetic-susceptibility effects. Magn Reson Med. 1988; 6:164-174. [PubMed: 3367774]

88. Williams DS, Detre JA, Leigh JS, et al. Magnetic-resonance-imaging of perfusion using spin inversion of arterial water. Proc Natl Acad Sci USA. 1992; 89:212-216. [PubMed: 1729691]

89. Donahue M, Lu H, Jones C, et al. An account of the discrepancy between MRI and PET cerebral blood flow measures. A high-field MRI investigation. NMR Biomed. 2006; 19:1043-1054. [PubMed: 16948114]

90. Heiss WD. Best measure of ischemic penumbra: Positron emission tomography. Stroke. 2003; 34:2534-2535. [PubMed: 12970510]

91. Warach S. Measurement of the ischemic penumbra with MRI: It's about time. Stroke. 2003; 34:2533-2534. [PubMed: 12970511]

92. Guadagno JV, Warburton EA, Jones PS, et al. How affected is oxygen metabolism in DWI lesions? A combined acute stroke PET-MR study. Neurology. 2006; 67:824-829. [PubMed: 16966545]

93. Takasawa M, Jones PS, Guadagno JV, et al. How reliable is perfusion MR in acute stroke? Validation and determination of the penumbra threshold against quantitative PET. Stroke. 2008; 39:870-877. [PubMed: 18258831]

94. Werner P, Saur D, Zeisig V, et al. Simultaneous PET/MRI in stroke: a case series. J Cereb Blood Flow Metab. 2015; 35:1421-1425. [PubMed: 26174332]

95. Rischpler C, Nekolla SG, Dregely I, et al. Hybrid PET/MR Imaging of the Heart: Potential, Initial Experiences, and Future Prospects. J Nucl Med. 2013; 54:402-415. [PubMed: 23404088]

96. Gonzalez RG, Fischman AJ, Guimaraes AR, et al. Functional MR in the evaluation of dementia correlation of abnormal dynamic cerebral blood-volume measurements with changes in cerebral metabolism on positron emission tomography with fludeoxyglucose F-18. Am J Neuroradiol. 1995; 16:1763-1770. [PubMed: 8693972]

97. Buckner RL, Snyder AZ, Shannon BJ, et al. Molecular, structural, and functional characterization of Alzheimer's disease: Evidence for a relationship between default activity, amyloid, and memory. J Neurosci. 2005; 25:7709-7717. [PubMed: 16120771]

98. Jack CR, Lowe VJ, Senjem ML, et al. C-II PiB and structural MRI provide complementary information in imaging of Alzheimers disease and amnestic mild cognitive impairment. Brain. 2008; 131:665-680. [PubMed: 18263627]

99. Villain N, Desgranges B, Viader F, et al. Relationships between hippocampal atrophy, white matter disruption, and gray matter hypometabolism in Alzheimer's disease. J Neurosci. 2008; 28:61746181. [PubMed: 18550759]

100. Sperling RA, Dickerson BC, Pihlajamaki M, et al. Functional alterations in memory networks in early Alzheimer's disease. Neuromolecular Med. 2010; 12:27-43. [PubMed: 20069392]

101. Catalano OA, Coutinho AM, Sahani DV, et al. Colorectal cancer staging: comparison of wholebody PET/CT and PET/MR. Abdominal radiology (New York). 2016

102. Moseley CK, Carlin SM, Neelamegam R, et al. An efficient and practical radiosynthesis of [11C]temozolomide. Org Lett. 2012; 14:5872-5875. [PubMed: 23151019]

103. Boujelben A, Watson M, McDougall S, et al. Multimodality imaging and mathematical modelling of drug delivery to glioblastomas. Interface Focus. 2016; 6:9. 


\section{KEY POINTS}

- Integrated PET/MRI scanners capable of simultaneous data acquisition are commercially available for human use.

- In addition to the hardware challenges that needed to be addressed for integrating PET and MRI, approaches to overcome specific methodological hurdles such as the need for deriving the attenuation maps from the MR data had to be implemented.

- Methodological improvements and the synergistic use of the simultaneously acquired datasets are expected to benefit numerous research and clinical applications. 


\section{SYNOPSIS}

Combined PET/MRI scanners capable of acquiring simultaneously the complementary information provided by the two imaging modalities are now available for human use. Although initial progress in the field was made more than two decades ago, it was the emergence of MR-compatible photon detector technology that allowed PET/MRI to move from the preclinical to the clinical arena. After addressing the hardware challenges for integrating the two imaging modalities, most of the efforts in the field have focused on developing MR-based attenuation correction methods both for neurological and whole-body applications, implementing approaches for improving one modality by using the data provided by the other one and exploring research and clinical applications that could benefit from the synergistic use of the multimodal data.

Magn Reson Imaging Clin N Am. Author manuscript; available in PMC 2018 May 01. 

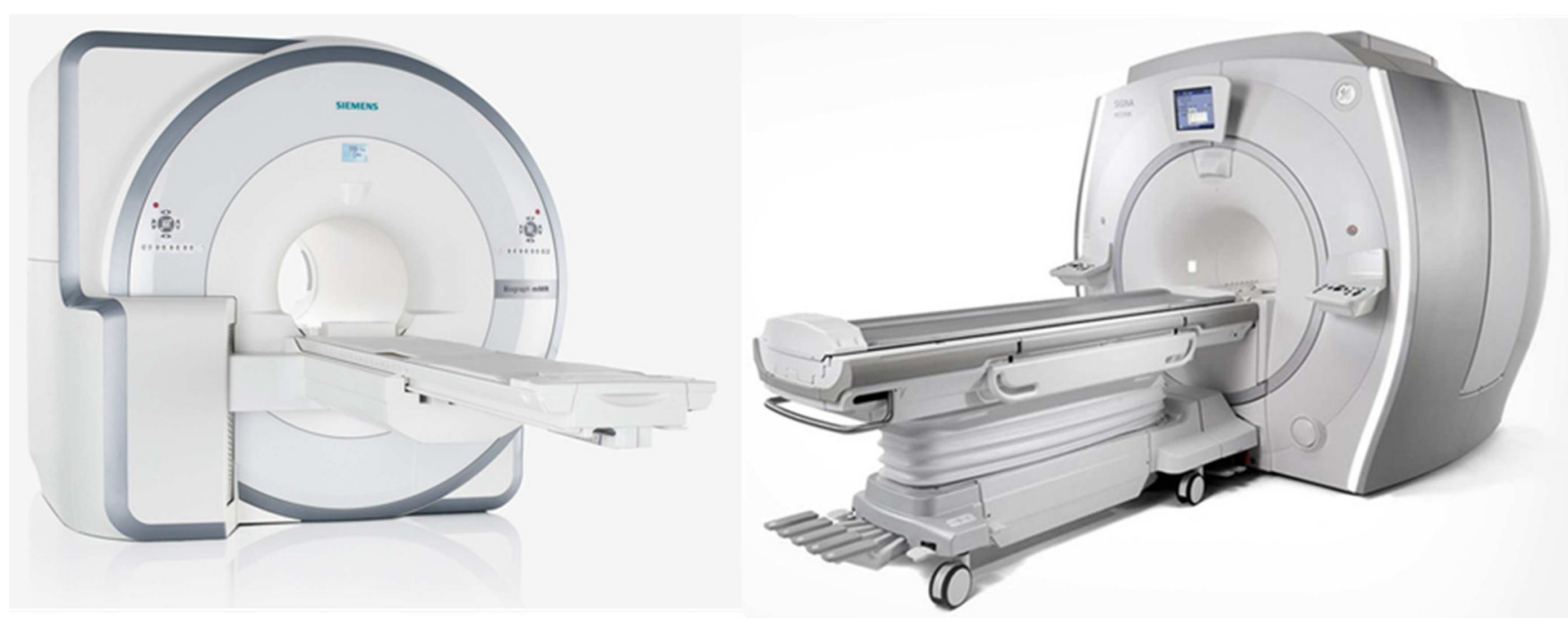

Figure 1.

Commercially available integrated PET/MRI scanners for human use: (left) Siemens Biograph mMR (picture downloaded from (2)) and (right) GE Signal PET/MRI (picture downloaded from (3)). From Siemens Medical Solutions, with permission. (https:// usa.healthcare.siemens.com/magnetic-resonance-imaging/mr-petscanner/biograph-mmr and http://www3.gehealthcare.com/en/products/categories/magnetic_resonance_imaging/3-0t/ signa_pet-mr. Accessed January 4, 2017). 


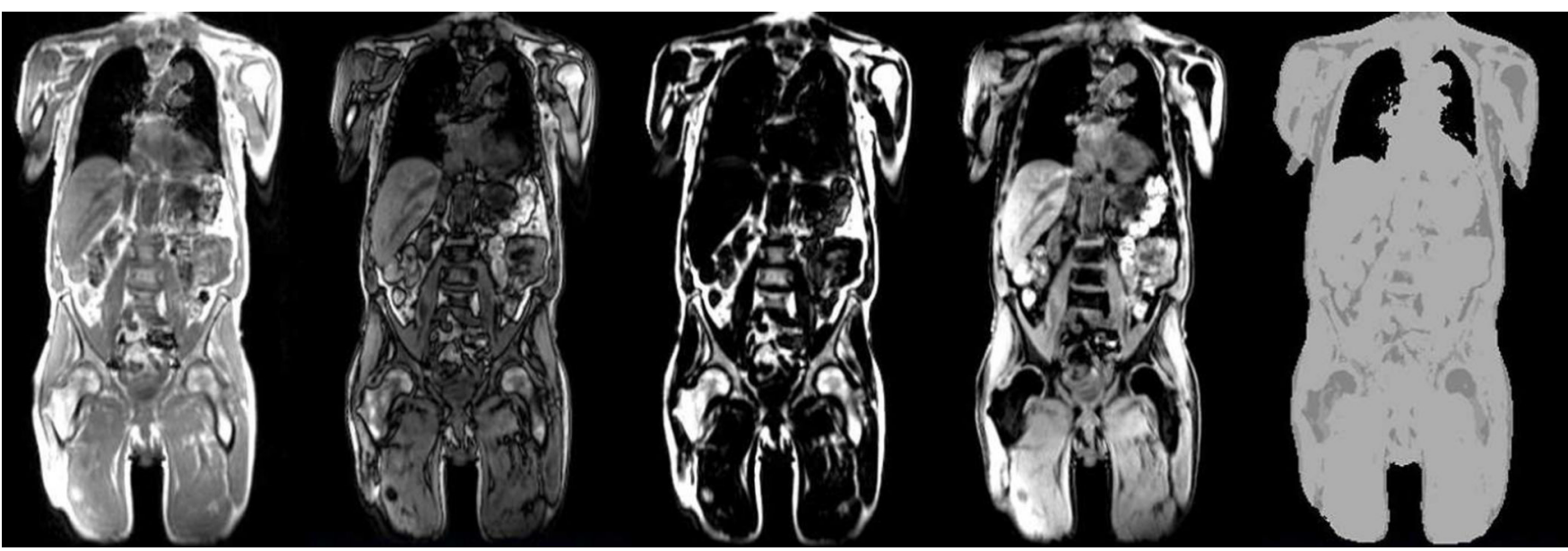

Figure 2.

Standard MR-based attenuation maps obtained on the Biograph mMR scanner. The in-phase, out-of-phase, fat, water MR images and the corresponding attenuation map generated from these images are shown from left to right. 

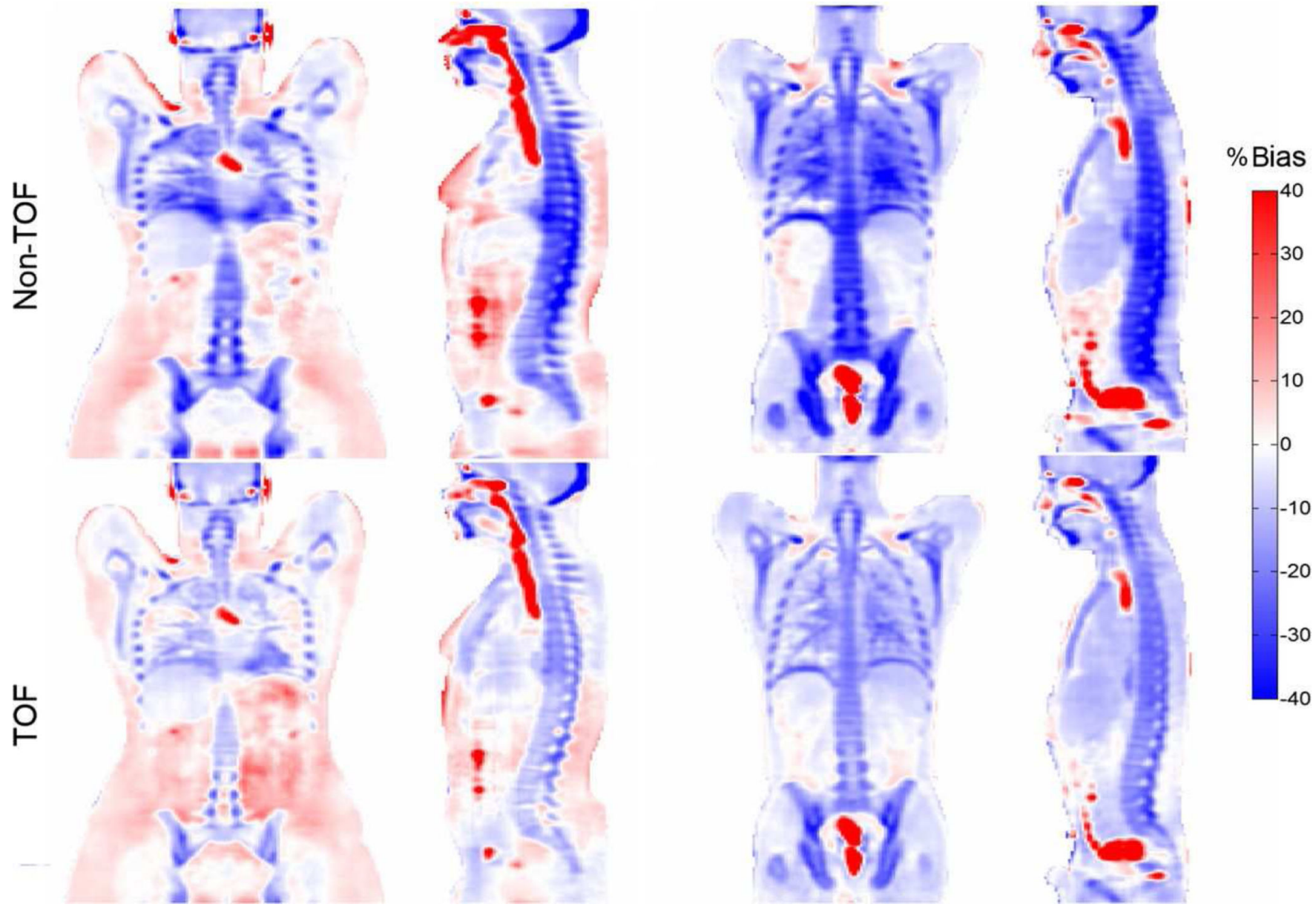

Figure 3.

Potential impact of TOF on the accuracy of the MR-based attenuation correction. The PET images attenuation corrected using a 4-tissue class MR-based approach obtained before and after including the TOF information in the reconstruction are shown for two representative patients (left and right panels). From Mehranian A, Zaidi H. Impact of Time-of-Flight PET on Quantification Errors in MR Imaging-Based Attenuation Correction. J Nucl Med. 2015;56:635-641, with permission. 

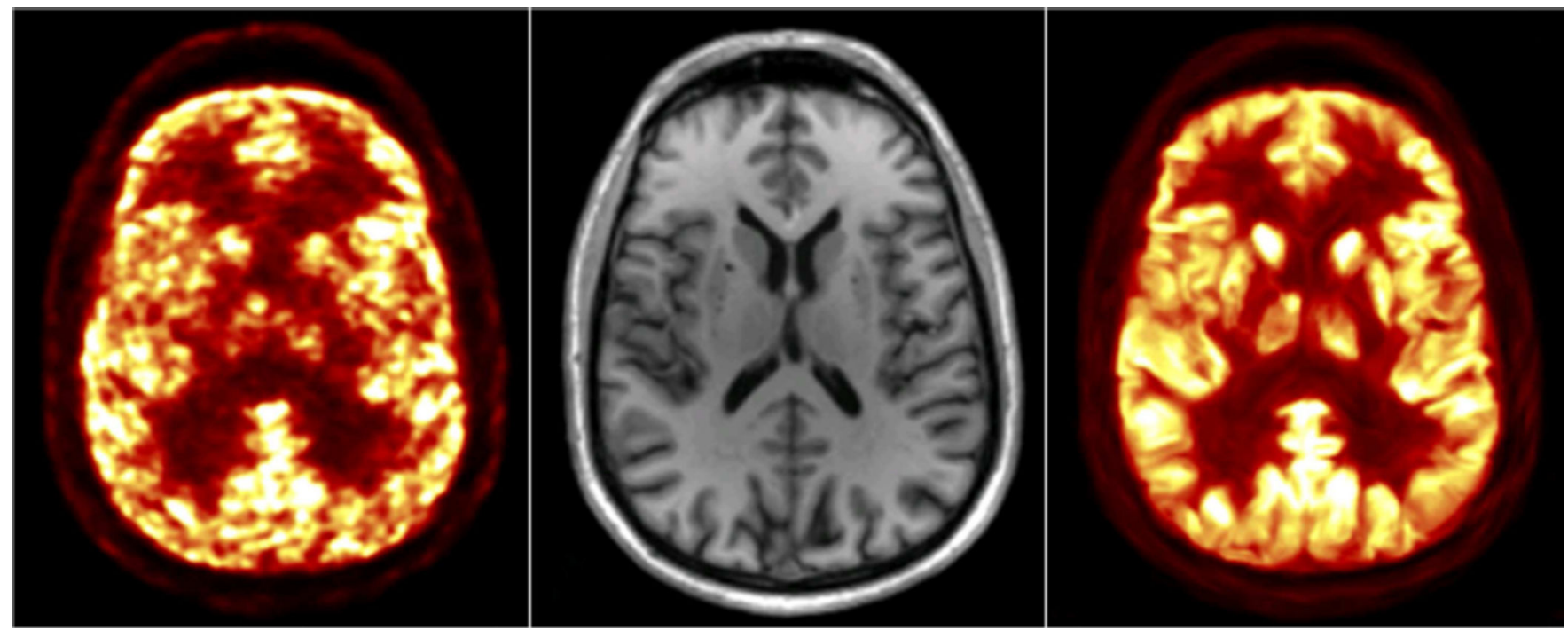

Figure 4.

Anatomy-aided PET image reconstruction: representative PET image reconstructed with a standard MLEM algorithm (left), the corresponding MR anatomical image (middle) and the PET image reconstructed using MR-derived priors derived (right). Courtesy of Kevin Chen, A.A. Martinos Center, MGH. 


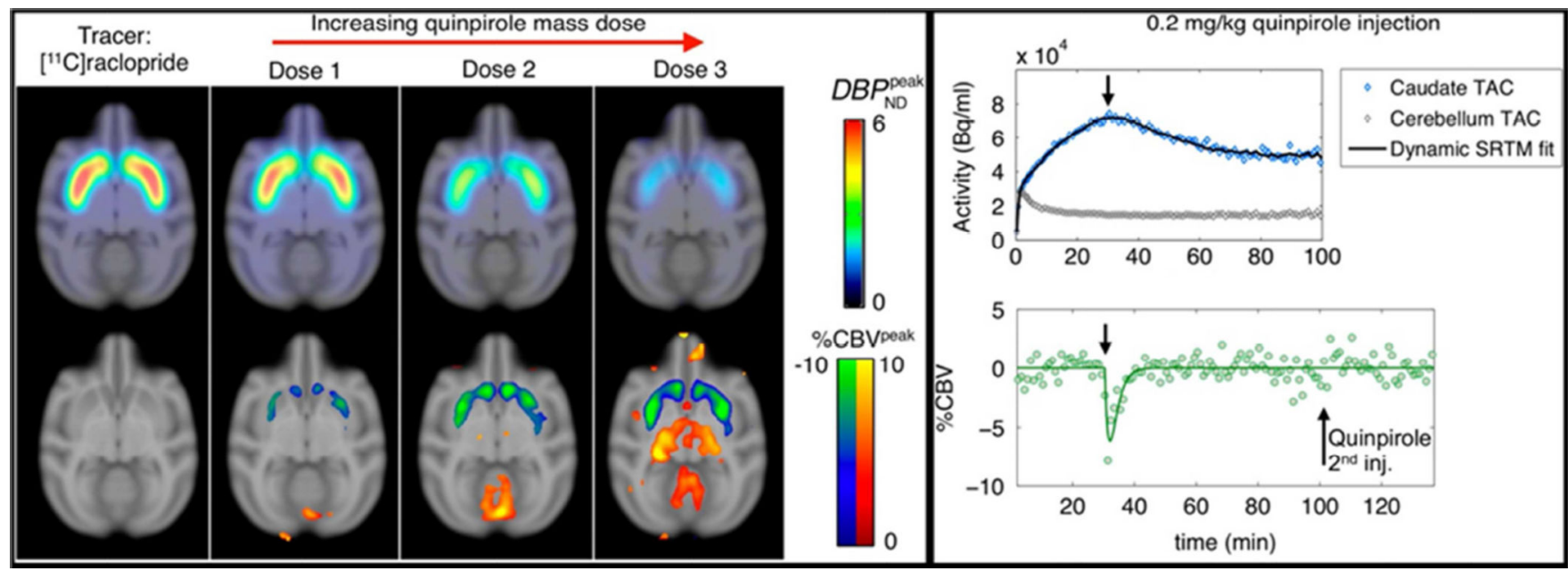

Figure 5.

In vivo simultaneous PET/fMRI study of the dynamics of dopamine receptor desensitization and internalization in non-human primates. Parametric maps from PET kinetic modeling and fMRI statistical analyses are shown on the left. The dynamic responses measured with PET and fMRI shown on the right demonstrate temporal dissociation between the two signals. From Sander CY, Hooker JM, Catana C, et al. Imaging Agonist-Induced D2/D3 Receptor Desensitization and Internalization In Vivo with PET/fMRI. Neuropsychopharmacology. 2016;41:1427-1436, with permission. 